\title{
Development of Simulation Platforms for Laser Phonosurgery, Laryngeal Endoscopy, and Fiber-carried Laser Procedures
}

\author{
Jan Alexeis C. Lacuata, MD, ${ }^{1}$ Abigail D. Sarmiento, MD $^{1}$ and Ryner Jose, D.C. Carrillo, MD, MSc (ClinEpi) $)^{1,2}$ \\ ${ }^{1}$ Department of Otorhinolaryngology, Philippine General Hospital, University of the Philippines Manila \\ ${ }^{2}$ Department of Anatomy, College of Medicine, University of the Philippines Manila
}

\begin{abstract}
Background. Simulators in laryngology are an essential part of training. They provide an avenue for medical students and resident trainees to practice valuable psychomotor skills outside the realm of an actual patient encounter thereby decreasing the risk of possible patient morbidity. Herein we present three locally manufactured simulation devices that can be used to train residents in laryngology procedures.
\end{abstract}

Objective. To present three simulation platform devices (Laser Box, Flexible Endoscopy Simulation, Thiel Cadaver Chair) and their applications in simulation-based learning

Methods. The Laser Box, Flexible Endoscopy Simulation, and Thiel Cadaver Chair were manufactured by outside parties designated as 'Laser Machinists'.

Results. Ten (10) units of the Laser Box, two (2) units of the Thiel Cadaver Chair, and two (2) units of the Flexible Endoscopy Simulation were constructed. They were used in a laryngology postgraduate course in our institution to teach the participants on endoscopies and laser phonosurgery.

Conclusion. Simulation for otolaryngologic procedures should be an essential part of training. The learning curve for procedures such as laryngoscopy and laser phonosurgery can be addressed with the production of simulation platforms. Most institutions in the Philippines still adopt the "see one, do one, teach one" approach, which lacks standardization and puts patients at risk. Various task trainers for laser phonosurgery and flexible endoscopy have been reported in the literature, but there seems to be no published data on the use of a cadaver chair for simulation. In the COVID-19 era, aside from being excellent teaching tools, simulation platforms derive their importance in helping train residents, educate medical students, and review consultants - maximizing skill development - and thereby decreasing repeated attempts, and indirectly, exposure to the SARS-CoV-2. Future validation studies are required for the models, with the eventual long-term goals to further standardize training, increase patient safety and incorporate a simulation-based curriculum for the specialty locally.

Keywords: Simulation Platform, Task Trainer, Phonosurgery, Laryngeal Endoscopy, Training Model

\section{INTRODUCTION}

There are numerous simulation models documented in the literature for the different subspecialties of otolaryngology. ${ }^{1,2}$ A systematic review by Javia et. al in 2011 revealed mostly temporal bone drilling and a few myringotomy simulators for otology; nasal endoscopy, endoscopic sinus

Corresponding author: Jan Alexeis C. Lacuata, MD Department of Otorhinolaryngology Philippine General Hospital University of the Philippines Manila Taft Avenue, Ermita, Manila 1000, Philippines Email: alexlacuatamd@gmail.com surgery and epistaxis simulators for rhinology; tonsillectomy and cleft palate repair models for the oral cavity; cricothyroidotomy simulators for procedures in the neck; and intubation, laryngeal dissection, and bronchoscopy models for laryngology. ${ }^{3}$ 
Our paper focuses on the subspecialty of laryngology, in which phonomicrosurgery has received special attention in the literature, with the development of various task trainers. As for the diagnostic skill set, the literature is scant with training models for flexible and rigid laryngoscopy simulation. Sheep ${ }^{4}$ have been utilized to train for flexible laryngoscopy of the pediatric airway, while a low-fidelity simulator involving a fenestrated ball inside a glove has been used to maneuver through the holes into the glove's fingers. ${ }^{5}$ Other institutions utilize mannequins but these are costly and are not easily available. The skills for laryngoscopy are commonly developed early on in residency training, with some patients becoming stepping stones to address the associated learning curve, at the expense of patient anxiety, discomfort, or trauma. ${ }^{4}$

Regardless of the blueprint, these simulation models aim to provide an avenue for medical students and resident trainees to practice valuable psychomotor skills outside the realm of an actual patient encounter - thereby decreasing the risk of possible patient morbidity.

Contag et. al echoes this in their paper, saying:

"The LDM (laryngeal dissection module) is designed to allow residents-in-training to develop fundamental phonomicrosurgical skills in an environment more forgiving of mistakes, while providing objective feedback and opportunities for basic skill set development." ${ }^{6}$

Consultants - even with their years of experience - can also benefit from simulation models. This is especially true for procedures that are uncommon or have been augmented in recent years by new technology, such as laser surgery for laryngology. ${ }^{2}$

We present three devices (Laser Box, Flexible Endoscopy Simulation, Sitting Cadaver Chair) conceptualized by our institution's Department of Otorhinolaryngology and their applications in simulation-based learning.

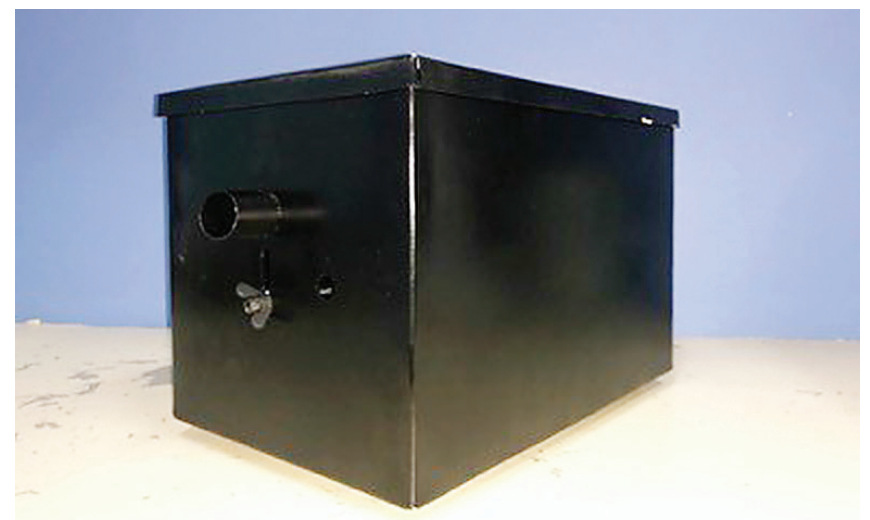

Figure 1. Oblique view of Laser Box Simulation Platform.

\section{MATERIALS AND METHODS}

\section{Laser Box Simulation Platform}

The Laser Box Simulation Platform or 'Laser Box' and its detachable cover were constructed with aluminum with an all-black finish (Figures 1 and 2). The color choice and lid help to simulate the low light conditions encountered during phonosurgery. The lid also serves to contain smoke within the device.

Inside the box is a metal platform, oriented predominantly in the axial plane, with a 'punched out' appearance (Figure 3). A wooden barbecue stick can be passed through one of the holes on one side, through a pig larynx, and then slipped to a contralateral hole, essentially providing additional fixation.

The platform is attached to a metal rod going through the center and midline of the box. The rod can be rotated in the clockwise and counterclockwise directions, adjusting the orientation of the platform, and therefore the laryngoscopic view of the vocal cords. The platform functions to cradle the porcine larynx (Figure 4).

There are two holes seen in the anterior aspect of the box (Figure 2). The first one is placed in the midline and center, slightly projecting anteriorly. It transitions into a laryngoscope - lip-like projection inside, just a few millimeters above the platform. The entire piece is meant to replicate a rigid laryngoscope used during suspension laryngoscopy for phonosurgical procedures - an opening for visualizing the laryngeal complex and for inserting microlaryngeal instruments. A suction tip can be inserted through this opening to evacuate smoke build-up (Figure 5).

The second hole is situated just below and to the right of the first hole (Figure 2). It serves to accommodate an endotracheal or laser tube, to be passed in between the vocal cords. The addition of the tube aims to replicate as close as possible the actual surgical view during phonomicrosurgery.

The box can be propped up on a wooden block for optimal visualization (Figure 6).

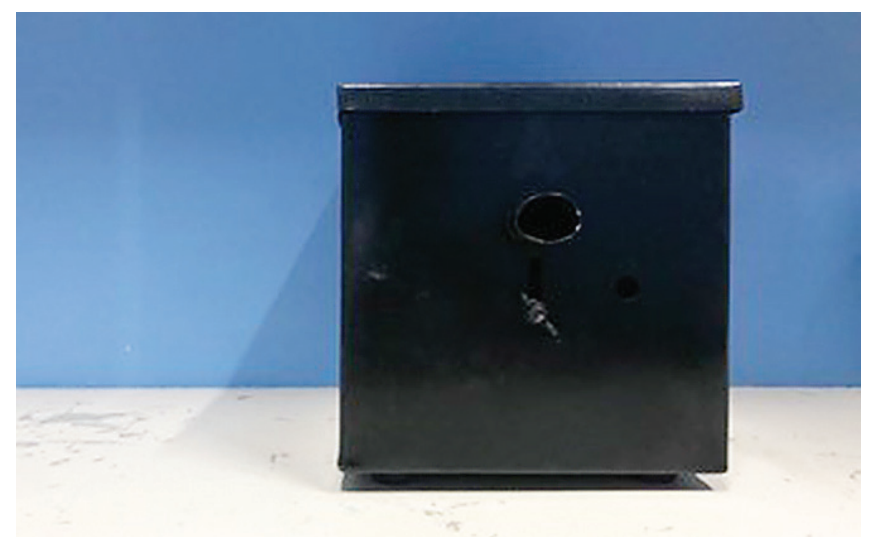

Figure 2. Anterior view of the Laser Box Simulation Platform. Note the presence of the two holes. 


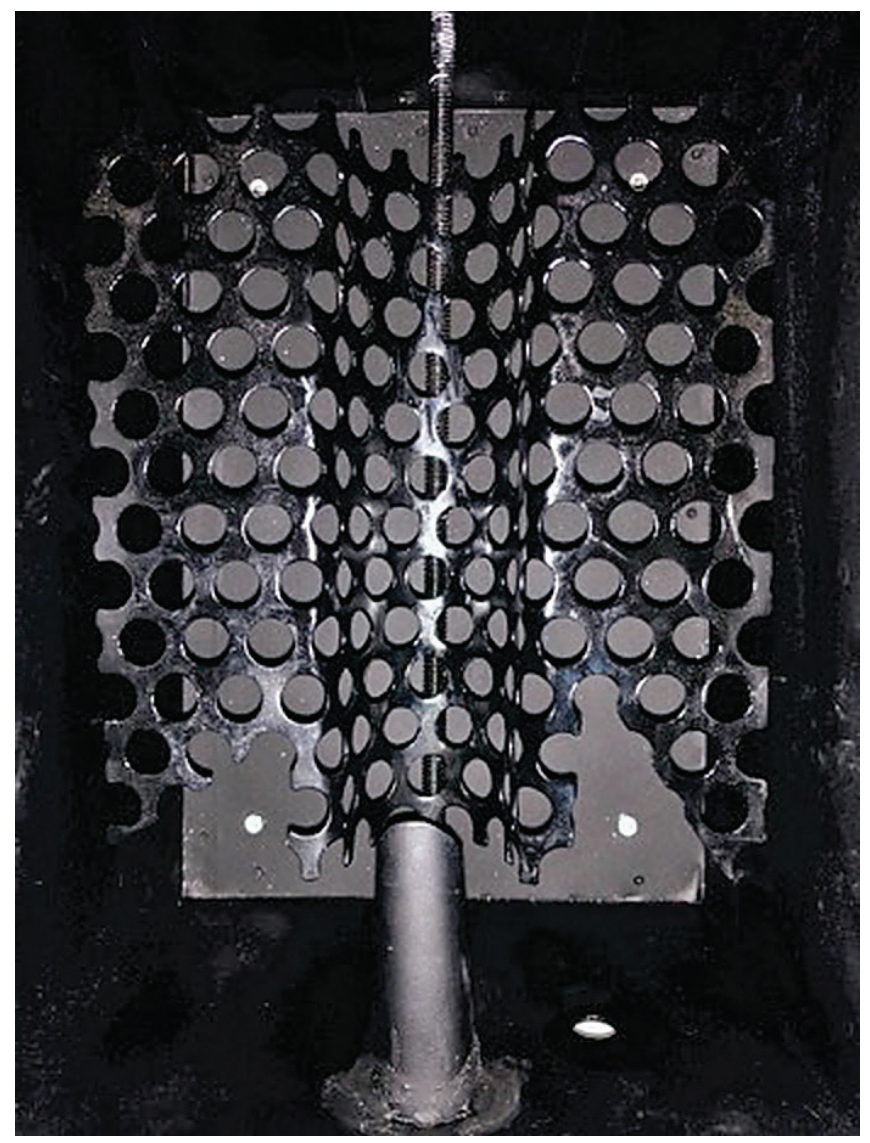

Figure 3. Bird's eye view of the box's interior. The metal platform spans the entire width of the box, and is studded with holes. There is a $U$ - shaped depression in the middle meant to cradle the porcine larynx. The laryngoscope lip is situated just above the platform.

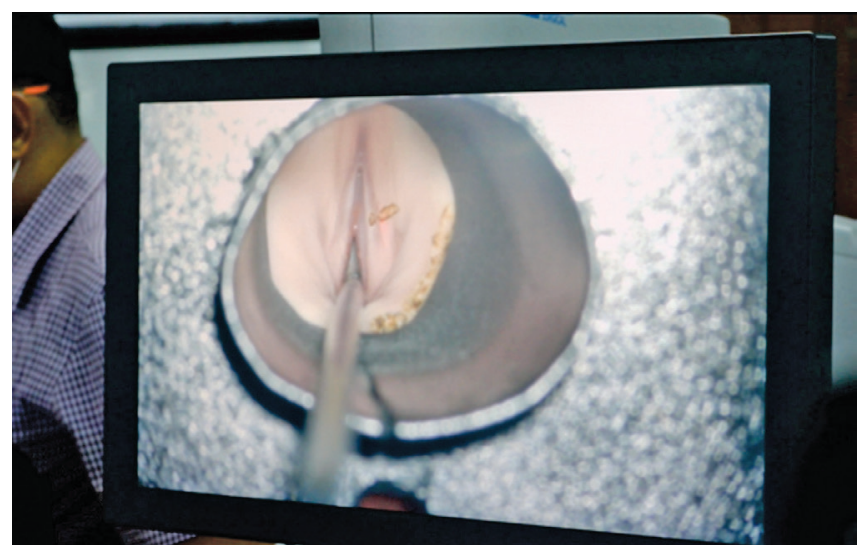

Figure 5. Operating microscope view through the box. Cordectomy is being attempted. Burn marks can be seen on the left true and false vocal cords. A suction tip is positioned between the vocal cords.

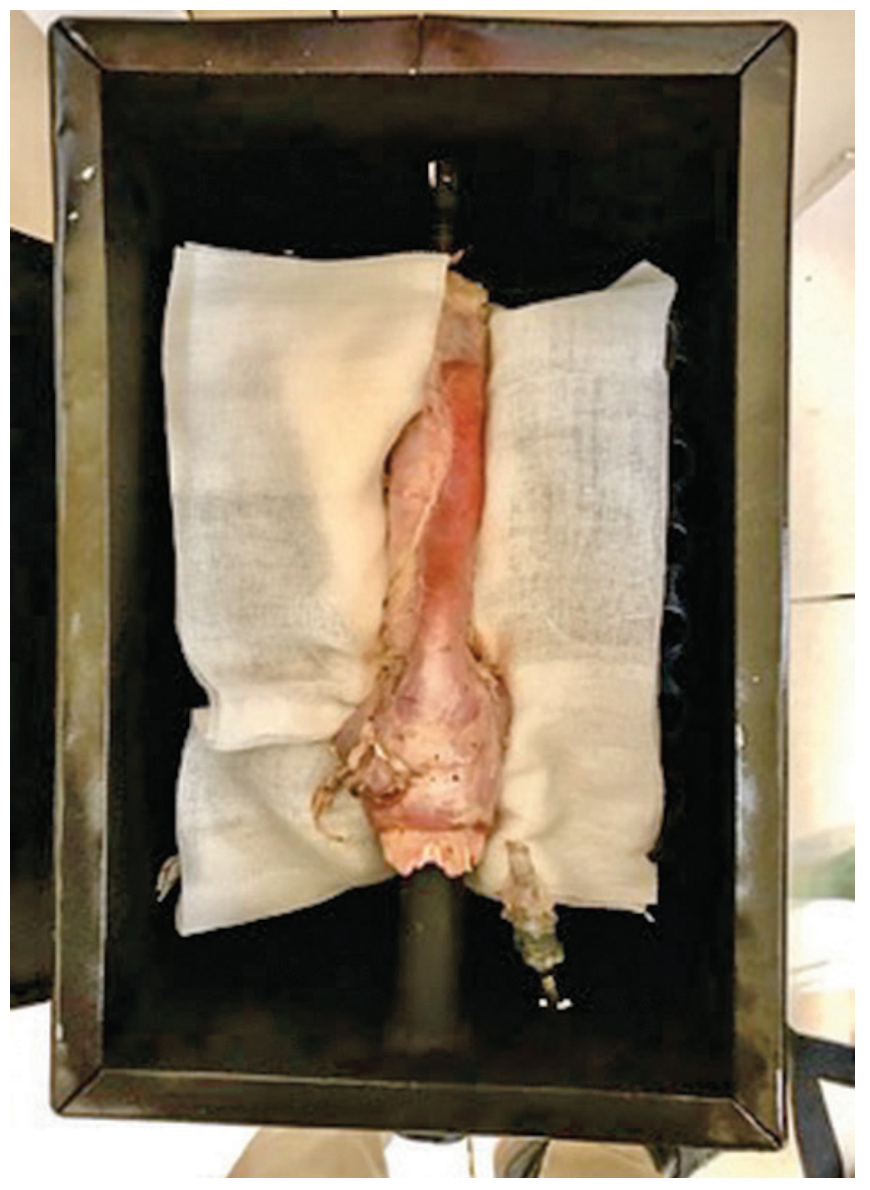

Figure 4. Bird's eye view of the box's interior. A porcine larynx is seen suspended in the midline. It hugs the laryngoscope lip. A laser tube cuff is seen to the right of the laryngoscope lip.

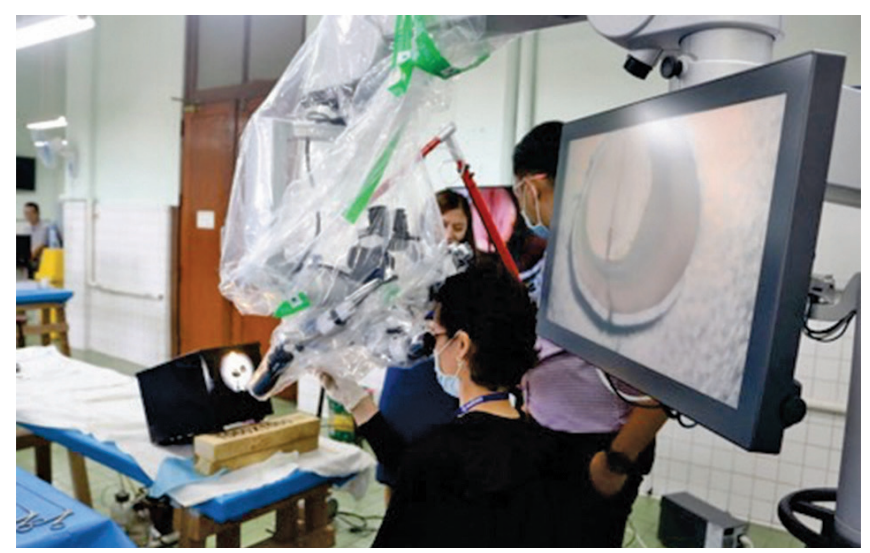

Figure 6. Set-up of the Laser Box and laser-mountable operating microscope. The box is propped up on a wooden block. The operator's view is projected onto the television screen. 
The dimensions of the box and its accompanying components are as follows:

- Box Height (with lid): $20.5 \mathrm{~cm}$

- Box Width: $20.5 \mathrm{~cm}$

- Box Length: $30.7 \mathrm{~cm}$

- Suspension Platform Length: $18.8 \mathrm{~cm}$

- Suspension Platform Width: $17.5 \mathrm{~cm}$

- Suspension Platform Bridge/Gap: $5.5 \mathrm{~cm}$

- Laryngoscope Hole Diameter: $3.2 \mathrm{~cm}$ across, $2.3 \mathrm{~cm}$ vertically

- Endotracheal/Laser Tube Hole Diameter: $1.3 \mathrm{~cm}$

\section{Thiel Cadaver Chair for Laryngoscopy, Phono- surgery, and Laser Simulation Procedure}

The Thiel Cadaver Chair for Laryngoscopy, Phonosurgery, and Laser Simulation Procedure - or 'Thiel Cadaver Chair' was constructed from wood, painted with water-resistant light grey paint (Figure 7). Wood was purposefully chosen due to its low cost and lightweight character. The authors deem that using metal, although feasible, would not be the most ideal; it is expensive and heavy. Furthermore, it will make the chair more prone to tipping over when the elevated center of gravity is taken into account. Only the side handles were constructed from metal for ease of carriage and transfer.

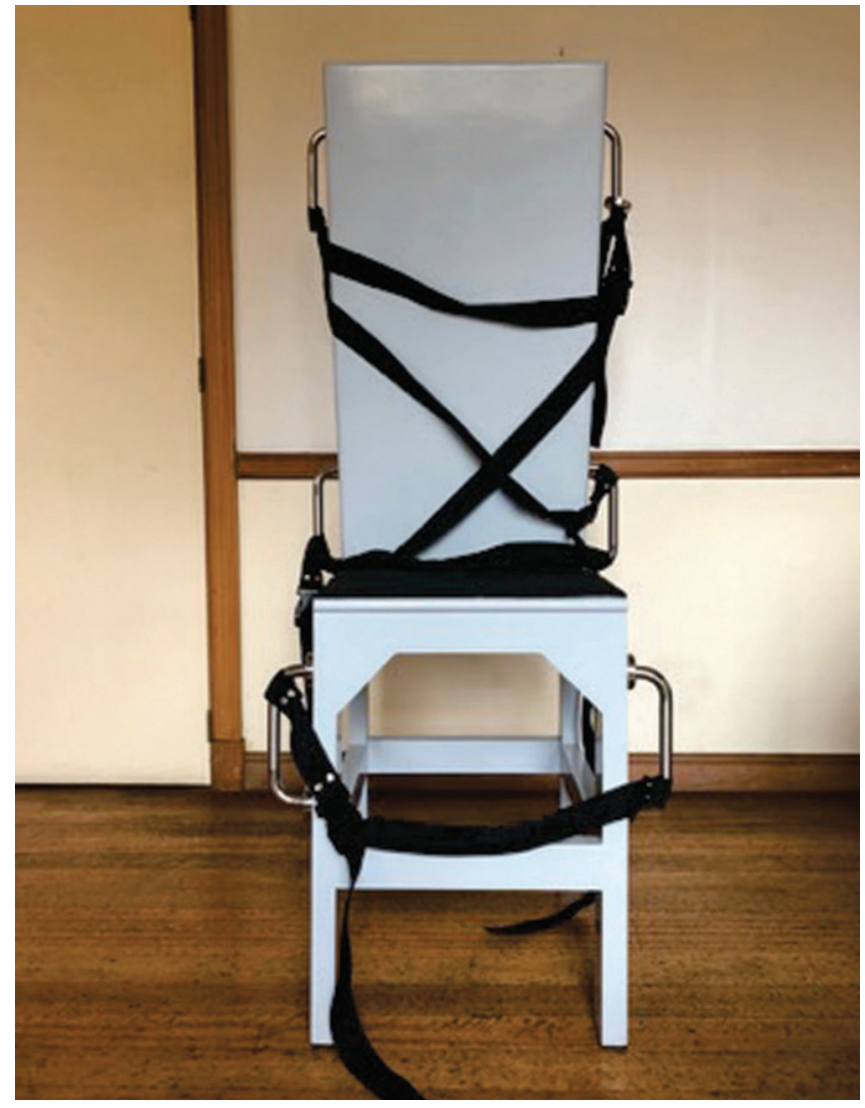

Figure 7. Anterior view of the Cadaver Chair.
The back part of the chair is movable, hinged at the bottom part of the chair's stiles (Figure 8). This provides weight against the cadaver's back, which will position the cadaver in slight hip flexion - essential to simulate the 'sniffing position', providing the best view of the laryngeal complex for laryngoscopic procedures (Figure 9).

The stiles have holes punched along them, in line with holes along with the movable back platform. Screws or rods may be passed along them to lock the back platform in a neutral position when not in use.

Attached along the stiles are four steel handles (two per side); there are also two steel handles (one per side) attached on the front legs of the chair. The handles facilitate the easier transfer of the entire unit while serving as posts through which black adjustable cloth straps can be attached.

The straps serve to hold the cadaver in place, across the chest and leg.

The dimensions of the chair are as follows:

- Chair height (top of stile to base of leg): $150 \mathrm{~cm}$

- Chair width: $45 \mathrm{~cm}$

- Chair length (anterior to posterior): $54 \mathrm{~cm}$

- Chair leg height: $64 \mathrm{~cm}$

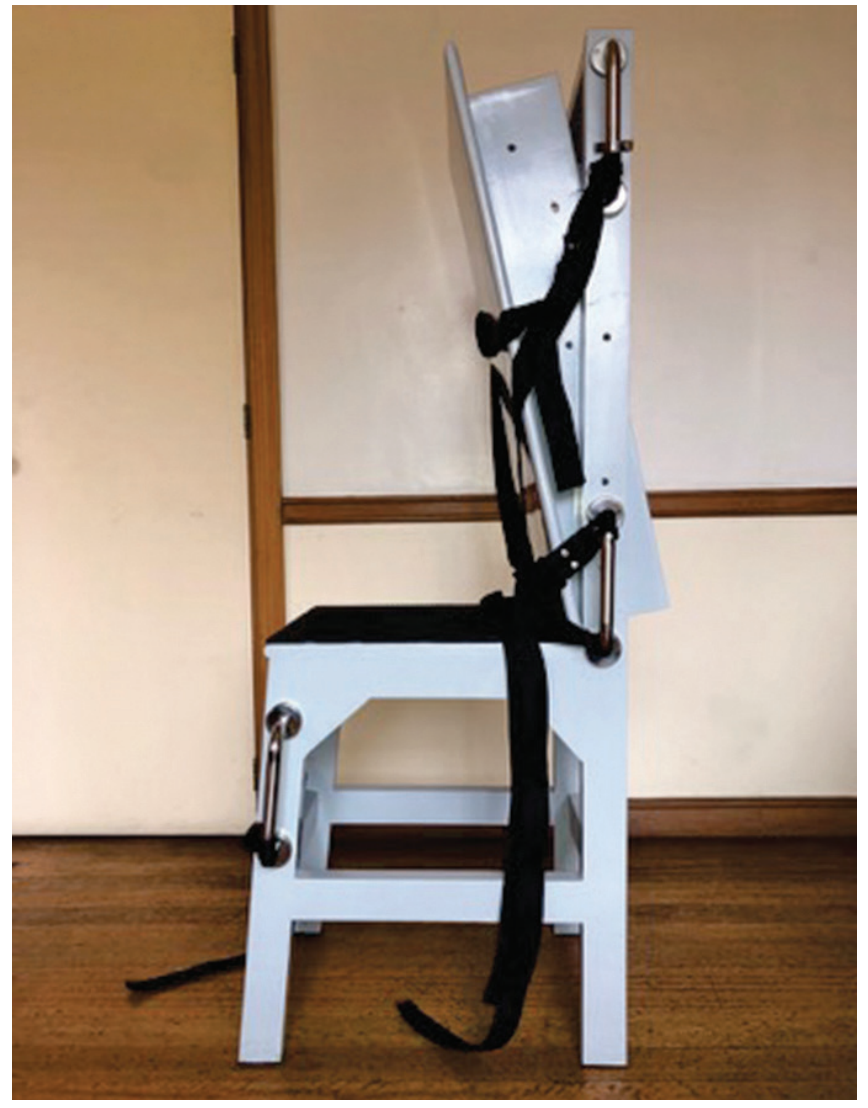

Figure 8. Lateral view of the Cadaver Chair. Note the angulated, adjustable back platform. 


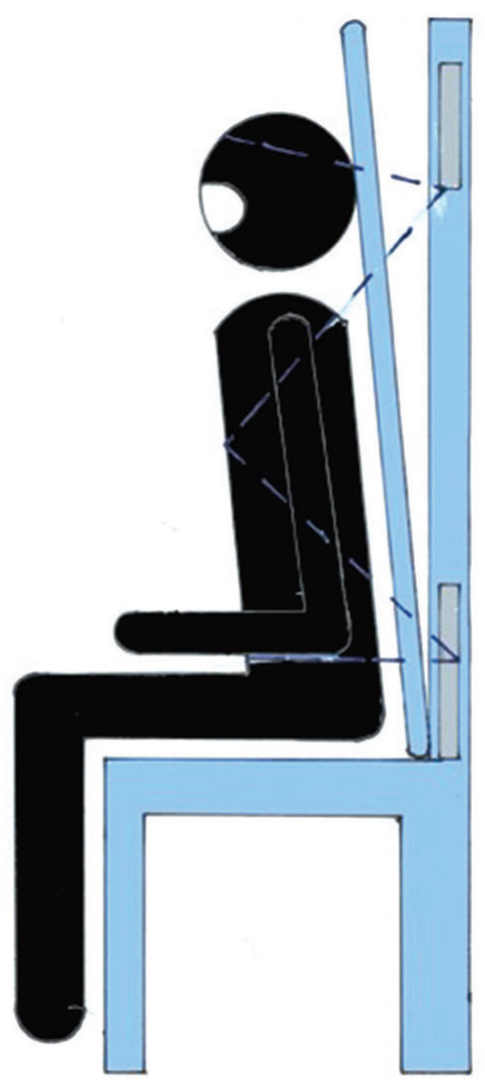

Figure 9. Schematic of the Cadaver Chair with a figure seated. Broken lines represent the straps across the head, chest and waist area. The back platform should position the cadaver in the sniffing position.

\section{Flexible Endoscopy Simulation Platform for Laryn- goscopic Procedures and Fiber-carried Laser}

The Flexible Endoscopy Simulation Platform for Laryngoscopic Procedures and Fiber-carried Laser or 'Flexible Endoscopy Simulation' was constructed from steel, painted with an all-black finish. Its structure is similar to the periscope of a submarine - a vertically-oriented cylinder curving forward at the top (Figures 10,11 and 12).

It features two circular openings anteriorly at the end of the curve, the smaller opening perched on top of the bigger opening. The smaller opening is divided in the midline by a steel black plate. This recreates the nasal cavity and the nasal septum.

The larger opening is meant to recreate the oral cavity. Together these openings converge into the body of the cylinder, representing the oropharynx and hypopharynx.

Below the larger opening are two small holes. A pliable, serrated, plastic lock can be threaded through the holes to hold the porcine/plastic larynx in place.

The cylinder is incomplete at the back, which provides access to the hollow interior of the device (Figure 13). The entire unit is mounted on a black rectangular base.

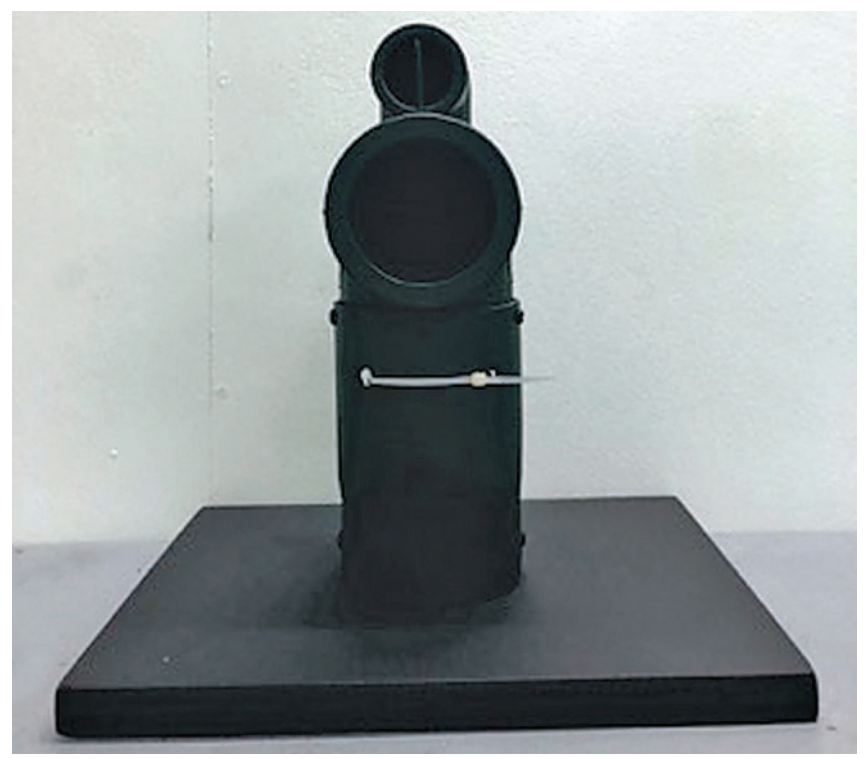

Figure 10. Anterior view of the Flexible Endoscopy Simulation platform. A pliable, serrated locking plastic is threaded through small holes below the larger opening. The plastic keeps the larynx inside in place.

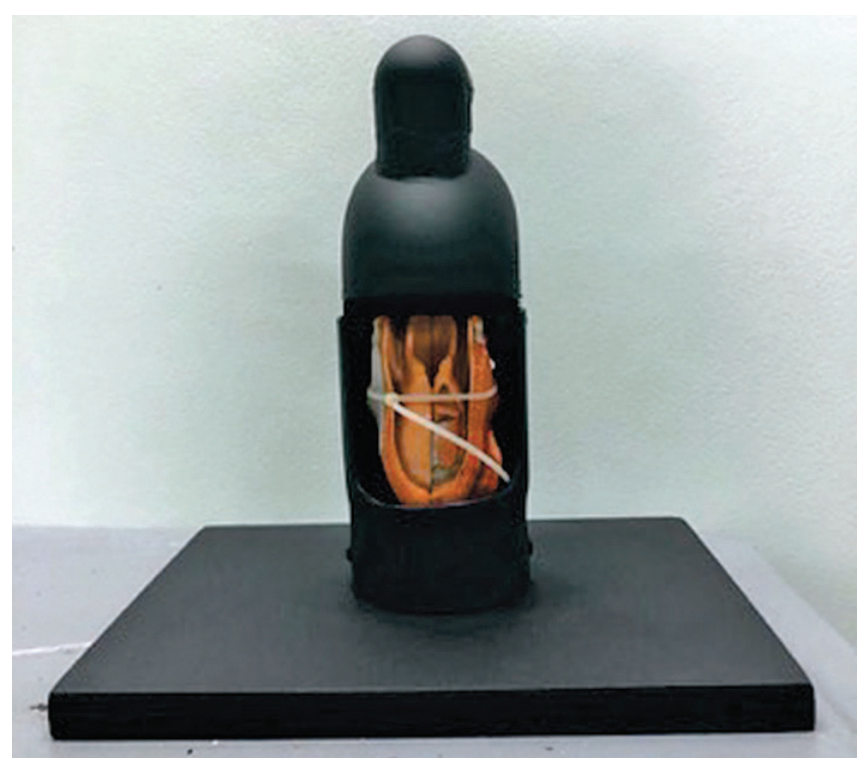

Figure 11. Posterior view. There is a plastic model larynx inside the platform, held in place by a locking plastic.

The dimensions of the platform are as follows:

- Height (from the base of cylinder to top of curved part): $25.5 \mathrm{~cm}$

- Rectangular base: $28 \mathrm{~cm}$ x $20.8 \mathrm{~cm}$ (length $\mathrm{x}$ width)

- Large circular opening - outer diameter: $8.1 \mathrm{~cm}$

- Large circular opening - inner diameter: $6.2 \mathrm{~cm}$

- Small circular opening - outer diameter: $4.3 \mathrm{~cm}$

- Small circular opening - inner diameter: $3.1 \mathrm{~cm}$

- Cylinder body diameter: $7.7 \mathrm{~cm}$ 


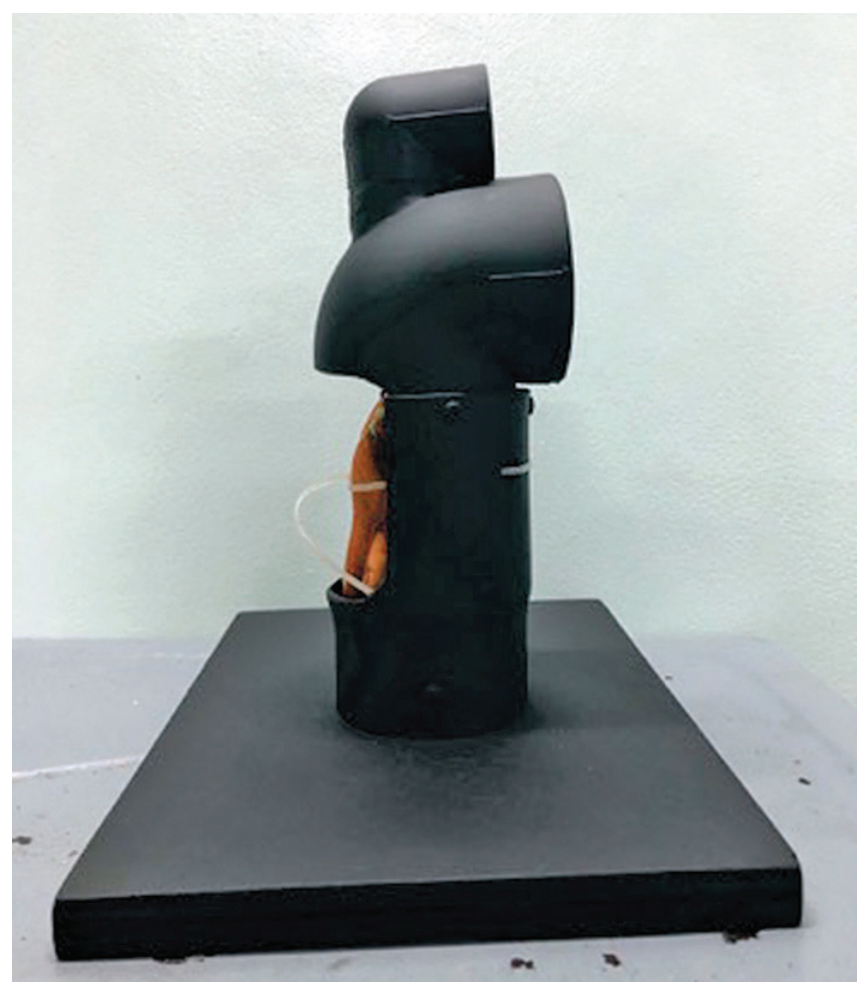

Figure 12. Lateral view. The model resembles the periscope of a submarine.

Two independent parties designated as 'Laser Machinists' were hired for the construction of the three platforms. The costs of the simulators (per unit) are as follows:

- Laser Box - P5,000.00 (around \$10)

- Thiel Cadaver Chair - 17,000.00 (around \$340)

- Flexible Endoscopy Simulation - P2,000.00 (around \$40)

\section{RESULTS}

Ten (10) units of the Laser Box, two (2) units of the Thiel Cadaver Chair, and two (2) units of the Flexible Endoscopy Simulation were constructed. They were used in a laryngology postgraduate course in our institution to teach the participants on endoscopies and laser phonosurgery.

The Laser Boxes were used for one session of phonomicrosurgery, utilizing a $\mathrm{CO}_{2}$ Laser. The Thiel Cadaver Chair found use in a simulation involving removal of a foreign body (medicine tablet foil) in the hypopharynx of a Thiel Cadaver using Frenkel forceps under rigid laryngoscopic visualization with a Berciwald scope. Flexible endoscopy was practiced using the Flexible Endoscopy Simulation. A plastic model larynx was housed within the platform.

\section{DISCUSSION}

To the best of the authors' knowledge, this is the only local paper reporting the construction of simulation platforms for training in otolaryngology. Common simulations imple-

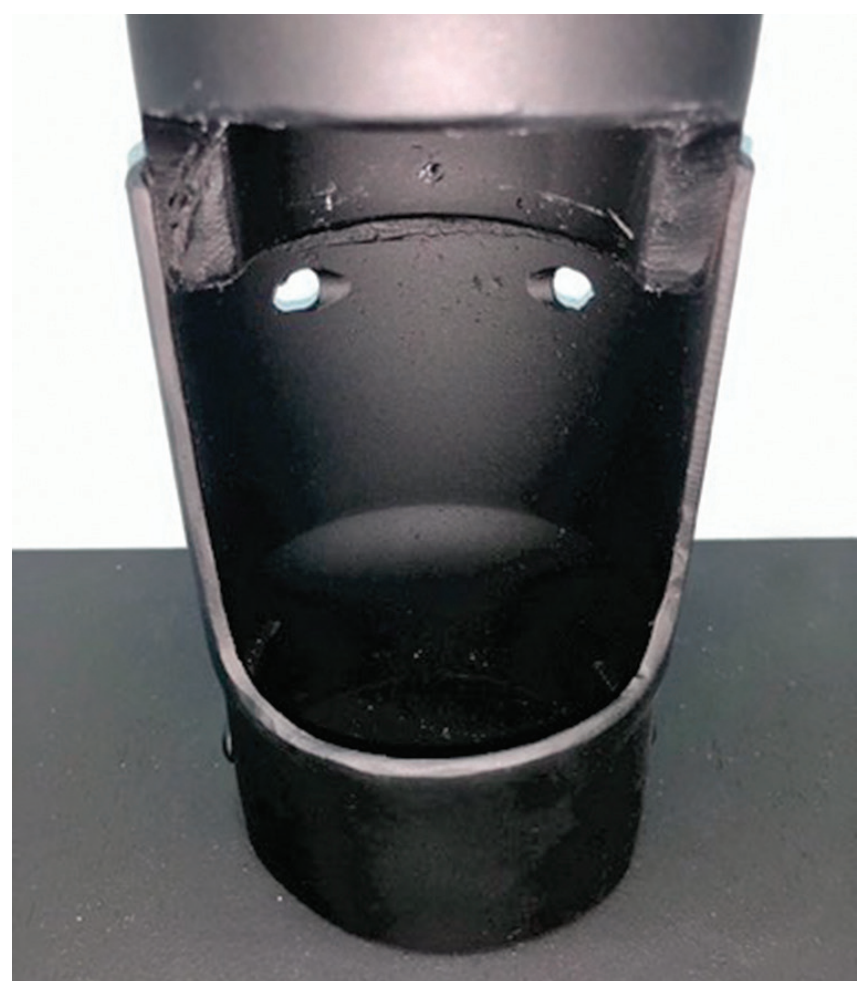

Figure 13. Posterior view, close-up. The model larynx is removed.

mented in medical schools include peripheral line insertion amongst each other and suturing sessions using pork. These types of simulations, although seemingly engaging for the budding medical student, is usually a one-time session, and therefore fares poorly in reproducibility. Psychomotor skills need to be practiced through repetition, and multiple training sessions cannot always be held due to economical and feasibility issues.

Current literature supports the use of simulation to teach procedural skills. Throughout much of residency training, the "see one, do one, teach one" has been repeatedly quoted. In their paper, Sawyer et al write:

"In this paradigm, procedural skill training is accomplished through direct patient care, with trainees practicing procedures on patients as part of a medical apprenticeship model. This training method has been brought under scrutiny within the past decade because of patient safety concerns, and an end to the "see one, do one, teach one" era through the use of simulationbased medical education, has been proposed."

A well-designed pilot study by Edmond evaluating endoscopic sinus surgery performance of otolaryngology residents after exposure to a virtual simulator showed a positive impact on surgical competency, with residents who were exposed to the simulator being rated better on scope control and dissection skill than those who weren't. ${ }^{8}$ Medical students who were trained with an intubation dummy 
showed faster intubation times and better success rates of the procedure on an actual patient, as opposed to those who were in the untrained group. ${ }^{9}$ The aforementioned studies were also reviewed by Javia et. al and were those with the highest levels of learning assessed (Level 3: Behavior, improved skills in real patients). No studies in the latter authors' systematic review reached Level 4 of outcomes-based measures (Level 4: results, patient outcome). ${ }^{3}$ Johnston et. al's randomized controlled trial demonstrated that simulation training using a high - cost and low - cost model for nasolaryngoscopy led to better patient comfort and endoscope manipulation during an actual patient encounter. Additionally, no difference was noted whether students were assigned to a high-cost or low-cost model, highlighting better reproducibility of the simulator across institutions. ${ }^{10}$ Smith et al. arrived at similar conclusions - flexible nasolaryngoscopy on a mannequin, combined with video feedback, resulted in better patient comfort scores, less time to reach the vocal folds, and overall performance scores. ${ }^{11}$ There seems to be a paucity of morbidity and mortality outcomes-based studies on the benefit of task trainers and simulators specific to otolaryngology - an avenue for further research. Nevertheless, the literature supports that simulation training across different subspecialties improves operator skill and competency.

In the following sections, we discuss the simulation platforms - where they stand with the currently available simulation models, and how they can contribute to a simulation-based framework for residency training in otolaryngology.

\section{Laser Box}

There have been various task trainers developed in the literature for microlaryngeal surgery. Porcine (pig) larynges, owing to their gross and histological similarity to human larynges, ${ }^{12}$ have been utilized, with the set-up of the task trainer modified. The pig larynx would be suspended in a wooden box, ${ }^{12}$ a steel-grade box, ${ }^{10}$ or incorporated in a mannequin model. ${ }^{13}$ Synthetic models for vocal cords were used in some prototypes - one using an industry-produced replica incorporated inside a mannequin, ${ }^{13}$ while another simply used a model larynx with a double-layered tissue paper on double-sided tape serving as the vocal cords. ${ }^{6}$ Focusing on cost and ease of reproducibility, grapes embedded within gelatin (with the grape skin serving as a faithful reproduction of the vocal fold epithelium) have also been conceptualized. ${ }^{14}$

Our model resembles Chan et al.'s ${ }^{15}$ Endoscopic Laryngeal Microsurgery Simulator (ELMS), using a pig larynx for the simulation. What makes our model different is the incorporation of an endotracheal/laser tube hole in the Laser Box, improving upon the fidelity of the device. Chan's model utilizes a square pallete as a holder for the larynx, which relies on tissue piled underneath for height elevation. The platform of our model is fixed at the midline and can be swiveled in the clockwise or counterclockwise rotation. Since our model's platform is suspended and has a "cradle" built to accommodate the organ, the larynx is more stable, even without fixation using sutures or ties. Should fixation be warranted, it would technically be less demanding, owing again to the structure of the platform, because the operators would just have to thread a single piece of barbecue stick, as detailed above.

The device has been tested during our laryngology course and the participants were able to do a laser cordectomy. Working with phonomicrosurgery, or any other type of surgery for that matter, we are naturally positioned to work at an angle, looking from above. Future iterations of our device could use a built-in standee at the front and bottom part of the box for angulation purposes. A notch could also be made in the interior laryngoscope lip to accommodate the tube, so as not to diminish the field of view of the operator.

\section{Thiel Cadaver Chair}

A literature search in the PubMed database, Cochrane database, www.herdin.ph using the keywords "chair", "cadaver", "simulation", "laryngoscopy", "sniffing position", "training" was done. None yielded relevant results about the use of a cadaver chair. Alternatively, a brute-force search method using a combination of the terms was also done via www.google.com but did not return related results. To the best of the authors' knowledge, this is the only current article describing a chair specially made to seat a cadaver.

Manikin simulators have been made to simulate training in the airway, but they are expensive, not easily accessible, and lack functional fidelity, despite attempts to improve their physical similarity to human subjects. ${ }^{16} \mathrm{~A}$ local study published by Villacorta et. al surmised that Thiel soft-embalmed cadavers were more life-like and "had the potential for research and practicing skills, such as endoscopy, laryngoscopy, and intubation". ${ }^{17}$ Another local study by Grullo et al. used soft-embalmed cadavers for surgical simulation of endoscopic sinus surgery, with the conclusion that the operation could be practiced with high fidelity and still present with the same technical difficulties. ${ }^{18}$

Other studies have utilized Thiel cadavers to train in fiberoptic tracheal intubation, ${ }^{19}$ facemask ventilation, direct laryngoscopy, and tracheal intubation, ${ }^{20}$ but none for upright procedures.

The key features of this setup are the chair and its adjustable back platform. The chair allows the cadaver to be seated, simulating the position and feel of an actual patient encounter. The back platform and head strap facilitate flexion of the hip and neck, placing the cadaver in a sniffing position, ultimately optimizing laryngeal exposure during endoscopies.

To use the chair, the operator simply has to position the cadaver in the upright position, with the cadaver's back against the swiveling backrest. The straps at the sides of the chair are used to maintain the cadaver in its position. Once in slight hip flexion - owing to the weight of the back platform - the cadaver's forehead is strapped to maintain its head in extension. 
During the postgraduate course, a foreign object was placed in the hypopharynx and the participant was made to remove it under direct visualization with Frenkel forceps. Although uncommon, a clinical scenario in which this will prove useful is the removal of a fish spine lodged in the hypopharynx. Rigid laryngoscopy is a common procedure that could be practiced on this setup by budding otolaryngologists-in-training.

Injection laryngoplasty could also be simulated, without the benefit of postoperative feedback from the patient, but more for the psychomotor skills that the resident needs to perform the procedure.

Although less feasible - and probably less practical in the current era of endoscopes - the set-up could also be theoretically used to teach medical students indirect laryngoscopy with a mirror. The learning curve for this procedure is higher than other physical examination techniques, and untrained medical students who encounter patients at the outpatient department more often than not end up eliciting the gag reflex due to poor technique.

With the Thiel Cadaver Chair setup, in theory, virtually any office-based laryngeal procedure can be practiced.

\section{Flexible Endoscopy Simulation}

Flexible laryngoscopy (also termed flexible nasolaryngoscopy, awake flexible laryngoscopy, flexible fibre-optic nasolaryngoscopy) is a basic competency that otolaryngologists have to learn early on in their training. Papers have been published detailing the improvement of this skill using mannequins with video feedback. ${ }^{11} \mathrm{~A}$ low-cost simulator using gloves and a fenestrated ball as aforementioned has also been utilized but with no apparent benefit. ${ }^{5}$

Our model structurally resembles the low-cost simulator constructed by Johnston et al., ${ }^{10}$ using pipe fittings, plastic pieces from a food container, and straws, with a few differences. The pipe fittings used in their model form a complete ring, while ours is deficient in the back. This makes it easier for the trainers to place a plastic model larynx or a porcine larynx to be visualized, as opposed to wedging artificial vocal cords within the body of our model. Their model replicated the inferior turbinates, structures that will always be encountered during the procedure, using a plastic straw, and the length of their "nasal floor" is longer than ours. Whether the faithful recreation of the nasal passages will have a significant impact on skill could be the topic of another study, we believe that the important part of the models is the curve that the participant will have to maneuver through. Chan et al., in the same paper, also fabricated an office-based laryngeal microsurgery simulator with a similar periscope-like apparatus on top of a box, then placed a porcine larynx on top. ${ }^{15}$

The main difference of our model from Chan's model, from a structural standpoint, is the presence of two openings anteriorly. The larger opening simulates the oral cavity/ oropharyngeal complex, providing future applications for simulation of procedures via the transoral route. Since the model was made to simulate flexible laryngoscopy procedures, we can also translate the utility of the model to train residents in fiber-carried laser procedures.

\section{The Value of Simulation Platforms in the COVID-19 Era}

Otolaryngology is a specialty that deals with multiple avenues for aerosolization of the SARS-CoV-2. Endoscopy in the nasal passages, laryngoscopy, and even a simple oral cavity examination are potential physical examination maneuvers that will pose a risk for transmission. As such, it has been recommended that procedures should be performed by the operator with the most skill, ${ }^{21}$ to ensure the least number of attempts at successful examination and thereby less exposure to untrained hands. From a training perspective, this will greatly decrease hands-on skills for beginning trainees, which is vital to the specialty.

Before the COVID-19 era, simulation platforms were a great adjunct to teaching and learning procedural skills. In their absence, one could still learn clinical skills, via the usual pedagogical platform which is to perform on an actual patient, regardless of experience.

Now, the authors think that the value of simulation platforms have been magnified because of the conundrum posed by this viral pandemic: how do you train otolaryngologists in procedures with a high risk of transmission, but at the same time minimize chances of failure - and indirectly - chances of exposure? We think that simulation platforms perfectly address this issue.

\section{CONCLUSION}

Simulation for otolaryngologic procedures should be an essential part of training. The learning curve for procedures such as laryngoscopy and laser phonosurgery can be addressed with the production of simulation platforms. Most institutions in the Philippines still adopt the "see one, do one, teach one" approach, which lacks standardization and puts patients at risk. Various task trainers for laser phonosurgery and flexible endoscopy have been reported in the literature, but there seems to be no published data on the use of a cadaver chair for simulation. In the COVID-19 era, aside from being excellent teaching tools, simulation platforms derive their importance in helping train residents, educate medical students, and review consultants maximizing skill development - and thereby decreasing repeated attempts, and indirectly, exposure to the SARS$\mathrm{CoV}-2$. Future validation studies are required for the models, with the eventual long-term goals to further standardize training, increase patient safety and incorporate a simulation-based curriculum for the specialty locally.

\section{Acknowledgments}

The authors are grateful to the current Chancellor of the University of the Philippines - Manila, Dr. Carmencita 
D. Padilla, MAHPS, the previous Dean of the University of the Philippines College of Medicine, Dr. Agnes D. Mejia, and her successor Dr. Charlotte M. Chiong, PhD, for their initial and continuing support for our simulationbased projects. We would also like to thank Mr. Reynaldo C. Rubio and Mr. Rosalito G. Artazo for the construction of the models; and Mr. Jupiter Kelly H. Baroa for the technical assistance for our projects.

\section{Statement of Authorship}

All authors participated in the data collection and analysis and approved the final version submitted.

\section{Author Disclosure}

The authors declared no conflicts of interest.

\section{Funding Source}

None.

\section{REFERENCES}

1. Thone N, Winter M, García-Matte RJ, et al. Simulation in Otolaryngology: A Teaching and Training Tool. Acta Otorrinolaringol Esp. 2017;68(2):115-20.

2. Deutsch ES. Simulation in Otolaryngology: Smart dummies and More. Otolaryngol Head Neck Surg. 2011;145(6):899-903.

3. Javia L, Deutsch ES. A Systematic Review of Simulators in Otolaryngology. Otolaryngol Head Neck Surg. 2012;147(6):999-1011.

4. Laeeq K, Pandian V, Skinner M, et al. Learning Curve for Competency in Flexible Laryngoscopy. Laryngoscope. 2010;120(10):1950-3.

5. Deutschmann MW, Yunker WK, Cho JJ, et al. Use of a Low-fidelity simulator to improve trans-nasal fibre-optic flexible laryngoscopy in the clinical setting: A randomized, single-blinded, prospective study. J Otolaryngol - Head Neck Surg. 2013;42(35):1-7.

6. Contag SP, Klein AM, Blount AC, et al. Validation of a Laryngeal Dissection Module for Phonomicrosurgical Training. Laryngoscope. 2009;119(1):211-5.

7. Sawyer T, White M, Zaveri P, et al. Learn, See, Practice, Prove, Do, Maintain: An Evidence-Based Pedagogical Framework for Procedural Skill Training in Medicine. Acad Med. 2015;90(8):1025-33.
8. Edmond C V. Impact of the endoscopic sinus surgical simulator on operating room performance. Laryngoscope. 2002;112(7 I):1148-58.

9. Howells TH, Emery FM, Twentyman JEC. Endotracheal Intubation Training Using a Simulator. Br J Anaesth. 1973;45(4):400-2.

10. Johnston DI, Selimi V V., Chang AA, et al. A low-cost alternative for nasolaryngoscopy simulation training equipment: A randomised controlled trial. J Laryngol Otol. 2015;129(11):1101-7.

11. Smith ME, Leung BC, Sharma R, et al. A randomized controlled trial of nasolaryngoscopy training techniques. Laryngoscope. 2014; 124(9):2034-8.

12. Nasser Kotby M, Wahba HA, Kamal E, et al. Animal model for training and improvement of the surgical skills in endolaryngeal microsurgery. J Voice. 2012;26(3):351-7.

13. Nixon IJ, Palmer FL, Ganly I, et al. An Integrated Simulator for Endolaryngeal Surgery. Laryngoscope. 2012;122(1):140-3.

14. Zambricki EA, Bergeron JL, DiRenzo EE, et al. Phonomicrosurgery Simulation: A Low-Cost Teaching Model Using Easily Accessible Materials. Laryngoscope. 2016;126(11):2528-33.

15. Chan CY, Lau DPC. Simulators and Models for Laryngeal Laser Surgery and Laser Myringotomy. Laryngoscope. 2016;126(9): 2089-91.

16. Kovacs G, Levitan R, Sandeski R. Clinical Cadavers as a Simulation Resource for Procedural Learning. AEM Educ Train. 2018;2(3): 239-47.

17. Villacorta PRF, Hernando HCT, Abdulla AJA, et al. A Comparative Study of Thiel Soft-embalmed and Formalin Preserved Cadavers for Anatomy Dissection. Acta Med Philipp. 2019;53(1):12-20.

18. Grullo PER, Hernando HCT, Carrillo RJD, et al. Surgical Simulation of Endoscopic Sinus Surgery using Thiel Soft-Embalmed Human Cadaver. Acta Med Philipp. 2017;51(1):24-7.

19. László CJ, Szúcs Z, Nemeskéri, et al. Human cadavers preserved using Thiel's method for the teaching of fibreoptically-guided intubation of the trachea: a laboratory investigation. Anaesthesia. 2018;73(1): $65-70$.

20. Szucs Z, László CJ, Baksa G, et al. Suitability of a preserved human cadaver model for the simulation of facemask ventilation, direct laryngoscopy and tracheal intubation: A laboratory investigation. $\mathrm{Br}$ J Anaesth. 2016;116(3):417-22.

21. Givi B, Schiff BA, Chinn SB, et al. Safety Recommendations for Evaluation and Surgery of the Head and Neck During the COVID-19 Pandemic. JAMA Otolaryngol Neck Surg. 2020;

\section{The Acta Medica Philippina is now accepting limited advertising for its front and back cover (colored), as well as for available spaces in some of its pages, as appropriate. For inquiries and submission of proposals, please email us at actamedicaphilippina.upm@up.edu.ph}

